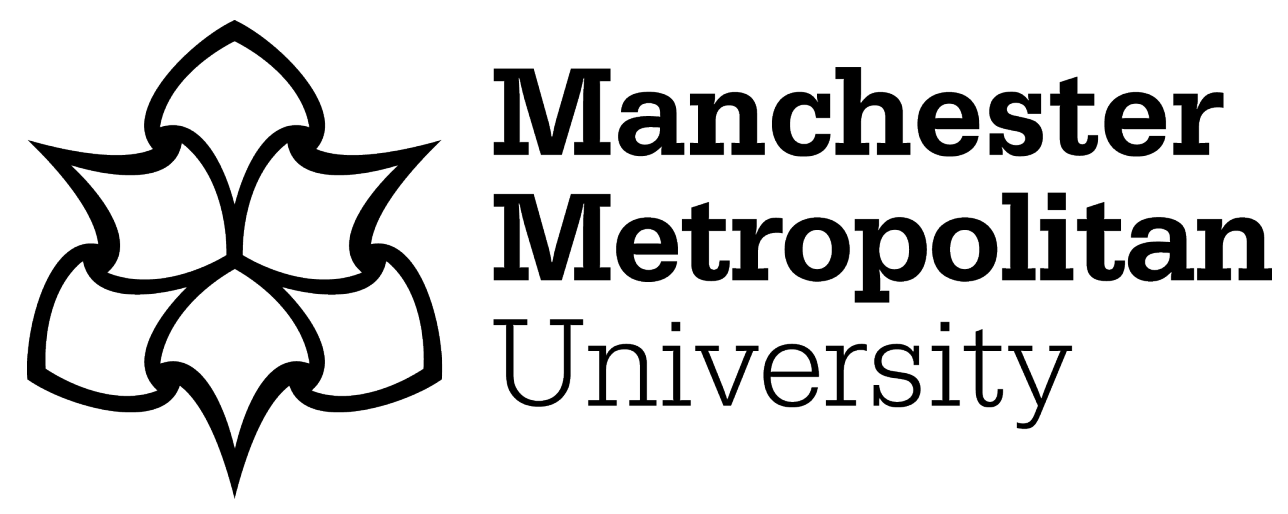

De Freitas, E (2017) Nonhuman findings from the laboratory of speculative sociology. The Minnesota Review, 2017 (88). pp. 116-126. ISSN 2157-4189

Downloaded from: https://e-space.mmu.ac.uk/625173/

Version: Accepted Version

Publisher: Duke University Press

DOI: https://doi.org/10.1215/00265667-3787450

Please cite the published version 


\section{Nonhuman Findings from the Laboratory of Speculative Sociology}

\section{The Laboratory of Speculative Sociology (LSS)}

The laboratory was designed and built in 2013, within the recently constructed Brooks building at Manchester Metropolitan University. Funding from the Centre for Biosocial Research on Learning and Behavior allowed researchers to design the laboratory with the latest biotechnologies and digital-synesthetic materials. A Finnish architect famous for his innovative but often awkward cyber-buildings was hired to produce the plans. During construction, he vanished and another team took over, altering the plans somewhat in order to use building materials recently developed in Guiyu, China, the electronic waste dump of the world.

The laboratory, now "complete," has been operating for four years. There are always at most three entryways into the laboratory and at minimum five exits. The shape, size, and location of both entrances and exits alter in response to the collective movements inside and out, and hence there are predictable patterns regarding where to find them. This ensures that there is an adequate sense of difficulty in entering but plenty of unexpected holes and tunnels for departing. When the number of entry points diminishes and the exits proliferate, the laboratory tends to empty. If exits proliferate without limit, the laboratory changes thermal state and inverts. Inversion involves radical changes in the environment, assimilation of the PAI (point at infinity), and eventually a highly unstable gaseous state that may, in theory, persist for years. Research findings during such periods are predicted to be highly informative if at all discernible. The lab is a dissipative system - $\mathrm{it}$ tends toward entropy. Like other living organisms, the lab itself is both energetic and informatic, and as such is sustained or dissipated through the ongoing alteration of energy gradients. After such dispersal and dissolution, a period of contraction and reassembling begins, always involving some recombinant permutation of actants, reproducing a laboratory that is always new but eerily familiar.

The Biosocial Centre was initially conceived as a transdisciplinary hub for advancing our understanding of human learning. A concerted effort to tap into contemporary scientific knowledge and new tech- 
nologies while avoiding rampant scientism has fueled center activities since its inception. The laboratory is not only or essentially a thermodynamic system but also a quantum event, by which all individuation of object or person is the effect of wave diffraction and temporal permutations. There is a time technician who micromanages this aspect, but he typically fails to coordinate participants, perhaps due to his overtraining or to the truly daunting task. Qualiants-which is the name given to those who attend and participate in laboratory activities - tend to collectively coordinate their movements through swarms and other immanent group structuring. Thus they manage to achieve forms despite the poor interventions of the technician.

The original objective of the laboratory was to study the flow of qualia and affect with/in an environment for which all such flow and pattern were immanent. Qualia were studied less as intrinsic internal feelings possessed by each qualiant ("what it feels like for me ...") and more as rumbling impersonal perturbations of the environment itself. These rumblings were disturbing and difficult to track, especially as they became almost imperceptible when a somewhat stable arrangement was actualized. During the first few years of operation, researchers found that the flow of qualia was fairly predictable, as long as they used rather simple parametric models. Their approach was initially modeled on Daniel Dennett's heterophenomenology, an approach to understanding consciousness that combined first-person accounts with scientific accounts, a combination of classic anthropology with the scientific method. The approach is to combine all data-scanning brains, blood flow, and other physiological and ecological information as well as interview data of a more "personal" flavor - in order to produce a more complete image of human subjectivity.

But the methodology was far too simplistic, coding the activity in terms of archaic quantifications ill-suited to the challenges of affect and nonhuman sentience. The interview data were always treated as symbolic and/or linguistic, which produced an overcoding of the events, as though all materiality was always saturated with meaningrather than some other effect achieved through such encounters. Findings during this period were still occupied with acausal models of perception, in which, despite attempts to think otherwise, reflection was the assumed operation at the heart of subjectification. This optical metaphor infiltrated even into earnest attempts to think the quantum, because light and visual patterns were still the source of theorizing the alternative optic metaphor of diffraction. Gradually, researchers turned to a more haptic conception of diffraction, one more truly based in the 
feeling of intensity when two waves meet. The brain scans, eye-tracking, and other physiological data were studied for how they converged and exhibited smooth manifold structures, using reliable optimization techniques for finding transition state triggers. Of course there remained correlations between the visual signal and the intense feeling of a wave peak, and participating qualiants recounted "infra-intense" experiences that corresponded with subject positions. For this reason, findings were oddly satisfying, confirming earlier reports regarding the power of affect in shaping identity.

Then in year three of the project, a more advanced real-time tracking technology was installed in the recycled floorboard fiber, which allowed researchers the wherewithal to study a darker phenomenology. This meant that the usual bias toward informatics (documenting signals of various kinds and interpreting these signals according to various hermeneutic theories) was radically overcome by an energetics that was - at that point - beyond the comprehension of research ethics protocols. Dark phenomenology was a way of studying energy gradients or contrasts, undermining the very premise of "experience" as that which is undergone by a unified "one" or "self," where the self was deemed to be a small and somewhat irrelevant bandwidth in the energetic system.

LSS researchers embraced a dark phenomenology that attended to the calculative affordances of the sensory confound. The new neural networks threaded into the laboratory floor were modeled on machine learning algorithms that had recently been made public at the NIPS conference. This meant that the algorithms were operating according to a kind of accelerated abductive reasoning (rather than inductive or deductive) and that the laboratory was at the forefront of an entirely new image of reason (Delanda 2011; Parisi 2016). This advanced computational infrastructure alone would have altered the daily working and future antics of the laboratory, but in addition there was a fluke weather formation that led to massive flooding across the north of England. The LSS was flooded, and its newly active floor achieved what no other speculative laboratory had achieved - precisely what Alexander Galloway and Eugene Thacker forecast in 2007: "In the future there will be a coincidence between happening and storage ... such that almost any space will be iteratively archived over time. . . . Space will become rewindable, fully simulated at all available time codes" (132). This new digitally and aquatically enhanced sensory environment was then able to bind multiscalar subjects (human and nonhuman) together in reconfigured modes of existence while 
transforming human experience from an agent-centered perceptual modality to a "worldly sensibility" (Hansen 2015, 197). Researchers at LSS were implicated in data mining below and above the time scale of the human, so that passive sensing was processed using new digital microsensors and powerful wet software.

The imperceptible aspects of the qualiant-environment became a source for quantification so that computational matter functioned as both remedy and poison for all that ailed the human sciences. Researchers rejoiced in what seemed to be a revolution in the study of "society" - a term now used simply to name a particular kind of assemblage sustained through coordinated movements. The infinitesimal valuations of unconscious encounters were calibrated using a new "qualitative probabilism" that tracked fractal differentials across the energy waves, putting into practice what Gilles Deleuze and Félix Guattari (1987) had gestured toward. Researchers watched as the affect circulated and contracted into knots of intensity. This immersion-approach, where the laboratory became a sort of soup or swamp of big data, forced the researchers to think differently about computational power. LSS was the first social science laboratory to move beyond prosthetic uses of technology, tied as these were to the notion of technology as an extension of the organism. Technology was no longer offering "scaffolding" for human activity, nor was it used to "off-load" tasks that might have previously been performed by hand. Qualiants who entered the laboratory were still asked to wear recording devices and plug themselves into various apparatuses, but the data "collected" during experiments were actually untraceable in these individual data sets. The laboratory itself was sensing the qualiants in other surprising ways, stretching and folding the discrete data points into a kind of fractal topology. Researchers at LSS were able to show how sensibility and quantification were not two different modes of being - they saw how quantification and computation inhered within every environment or materiality and how even our most intimate impulses were deeply computational, although not scripted. Thus the digital aspect of the laboratory was as "live" as any other aspect, which meant that the entire qual-quant distinction was subtended and the technology was truly immersive.

LSS researchers realized that they would need a new "climatology" of the social, following the suggestion of Galloway and Thacker (2007). In many ways, the laboratory was an expression of a certain agony that had taken hold of the social sciences during the previous century. That agony concerned the very concepts of the social and the 
individual, both utterly under siege during the latter half of the twentieth century. Influenced by a take-up of the nature philosophy of Alfred Whitehead, Mark Hansen (2015) had proposed that the best approach to computational media was to develop ways of studying the expanded sensory contact that characterized our new "worldly sensibility." These pronouncements, however, were mere fantasy until the experiments at the LSS in Manchester. Experiments moved beyond "perception studies" to a complex intervention into a more-than-human sensibility.

When twelve qualiants entered the laboratory in July 2016 during an experiment focusing on movement and noise, researchers suddenly felt a palpable change in the lab sensibility. They waited for four days before a single signal was detected - that strange, almost unrecognizable signal was evidence that the qualiants were no longer twelve, but seemed to have become a one with near infinite heterogeneous vibratory power across their signaling surface. Director Miriam Foost declared, "We are surely in the midst of a re-engineering of life itself, where a proliferation of non-perceptual sensible data feeds-forward into possible futures."

\section{The Nonhuman Side of Social Science}

The LSS is a work of speculative fiction. Speculative fiction helps us imagine how we might do social science differently in that it helps us interrogate the very concepts of "social" and "science" and offers an alternative model for how they might be reassembled. The LSS is an example of how social science can become unrecognizable to itself through art and fiction. Such work puts flesh on a thought experiment and does philosophy through character and story. The "speculative" in speculative fiction plays with the apparent fixity of the material world and explores fundamental philosophical questions about consciousness, time, life, and the nonhuman. The present historical moment, with its particular sociotechnical aspect, is used as a base for extreme extrapolation, producing another world that is weirdly and shockingly possible. The laboratory depicted above is in fact a distorted version of a planned laboratory focused on sense and affect, also located in Manchester. My writing of this speculative fiction was a method for opening up the weird future of the actual laboratory in Manchester. Following David Roden (2014), I was looking for a way to not preemptively curtail the weirdness of the posthuman, using fiction to help me imagine a new empiricism that might be taken up in the actual laboratory. 
In the example of the LSS, individual actions and actors are enmeshed in the water-soaked neural network algorithm threaded through the laboratory floor and are thus plugged into the recycled materials from the digital waste dump. The laboratory itself becomes a stochastic swarming that belies the very idea of individual (and hence the twelve become one) while sustaining an irreducible heterogeneity. As a form of science fiction, the method involves extrapolation and fabulation, often focusing on the limits of science and technology and on the spatiotemporal configurations of embodiment. Just as science and technology seem to evolve, or at the least mutate, so does our spatiotemporal framing of life and being. Speculative literature asks the "what if?" question and twists the imagined future just enough to make us wonder about how life could be different. This can involve taking a simple characteristic of this world and continuously stretching and distorting it until it becomes almost unrecognizable but is clearly an evolution or involution of the original.

The LSS is meant to show how we might study sensation and perception differently, how current technological tools are changing the very idea of what sensation is, where perception occurs, and whether sensory capacity might be reconceived in nonhuman terms, or at least beyond the concept of the individual who processes sensory information in order to make perceptual judgments. The laboratory is alive with computational capacity, recursively elaborating whatever "data" are generated, so that it is no longer simply a container in which activity is recorded. The very notion of recording data is radically reconceived through this speculative fiction. If this is plausibly a new image of empiricism, it is also a continuation of scientific practice. Bruno Latour (2005) and Isabelle Stengers (2000) have shown us how speculation plays a significant role in all inventive scientific practices. While the linguistic turn in the social sciences seemed to banish the material world from the realm of study, declaiming that we are always trapped within language, scientists working in the "physical" sciences have carried on speculatively imagining a nonhuman world rich with agencies. Rather than treat matter as inert and passive, "scientists work by negotiating with nonhuman entities, and by entering into alliances with them" (Shaviro 2015, 11). This perspective on science, fully elaborated in science and technology studies (STS), is beginning to influence the practice of social science through the work of Karen Barad, Bruno Latour, and Vicky Kirby, who turn to the practice of science and the physical world for insights into how we might do social science differently. 
Speculative sociology might be contrasted to what Latour calls "critical sociology," which he argues is committed to the dangerous game of social engineering, because it refuses to study the social as that which emerges. The critical sociology - typically associated with Pierre Bourdieu - that has come to dominate the social sciences assumes a particular concept of the social in advance and refuses to recognize the radical ingenuity of life to create a radically different kind of social. A speculative approach will instead pursue a sociology of associations, allowing the very concept of the social to mutate and emerge and become perhaps totally unrecognizable. The test, suggests Latour, for gauging the quality of a sociology of associations might be: "Are the concepts of the actors allowed to be stronger than that of the analyst, or is it the analyst who is doing all the talking?" In other words, does your social science come ready-handed with its coding devices - terms like habitus, cultural capital, or even rhizome-so that science becomes only the coding of empirical data? Instead, the speculative sociologist surrenders to the material force of the unknown and is overcome with monstrous agencies. This is not fiction in the service of metaphor but rather fiction that pursues the monster. The monstrous is a more radical break with current practice and a much bigger risk than the making of a metaphor.

This kind of speculative philosophy requires imagining otherwise - quite literally imagining the thought and behavior of the nonhuman, which is precisely what literature does so well. Latour claims that "it is only through continuous familiarity with literature that ANT sociologists might become less wooden, less rigid, less stiff in their definition of what sort of agencies populate the world" (2005, 55). Sociologists of associations seek innovation and controversy, and Latour is keen to point to the potential of art in doing just that: "Finally, when everything else has failed, the resource of fiction can bring - through the use of counterfactual history, thought experiments, and 'scientification' - the solid objects of today into the fluid states where their connections with humans may make sense. Here, again, sociologists have a lot to learn from artists" (82). The difference between this sociology and previous sociologies is the attempt to center the more-than-human in the analysis. Of course sociology has always been interested in how people live in relation to nonhuman objects - be it the material conditions of a geographic site or the particular media and technology at work in a culture - but most often these are treated as carriers or vehicles of human meaning. "Like humble servants, they live on the margins of the social doing most of the 
work but never allowed to be represented as such. There seems to be no way, no conduit, no entry point for them to be knitted together with the same wool as the rest of the social ties" (73).

We see here that Latour aims to generate research reports that recognize or incorporate non-human agencies as central players. These are actants that are as real as any others, but they are massively distributed across large networks of association, and it is challenging for more confined actants to recognize them as "objects." Through this empirical and practical approach to the materiality of life, Latour aims to show that objects do not simply express human power relations, symbolize social hierarchies, reify gender inequality, reinforce culture, and so on, for all such ways of speaking treat the objects as the carrier of human meaning.

The LSS researchers in the fiction above begin with Dennett's (1991) "heterophenomenology," but they soon realize that the interview data overcode all the other data in terms of discursive "meaning." Findings seem to support what they have always already known, and their attention to language use as meaning making blinds them to the other ways that language is coupled materially to the environment. Thus the LSS becomes stuck - much as social science has been stuck - treating language as the total environment and failing to encounter anything outside it. The challenge for the LSS researchers is to examine the material coupling of speech and other language acts with other materialities that operate outside a communication model. In the case of LSS, this is achieved only through the threading of new materials (assembled from recycled e-waste) and the haphazard introduction of floodwaters, which are also laced with the toxic traces of Manchester's industrial history. In this way, the new comes into being.

Social science must become a kind of nonhuman sociology infused with ecological insights and operating within the perspective of the Anthropocene.

\section{Body, Touch, and Environment}

When we study sensory experience, we are conventionally interested in how percepts and affects contract into bodies. Those bodies are then organized into sensory-motor agents. Perception is a word that gets us trapped into imagining a willful activity that occurs in the individuated mind of the body - as though the body were processing information. The challenge in sense studies is then to think about perception as an event that animates particular environments in particular ways, distributing the sensible unevenly across an environment. 
This speaks to the biopolitical issues pertaining to sense studies, and it speaks to the question of responsibility.

Findings from the laboratory of speculative sociology force us to ask how the politics of aesthetics changes in computational cultures. If it is the affective nature of aesthetics that is studied in the LSS, then findings suggest that the embodied nature of sentience is not confined to humans; sentience is achieved through individuation, true, but through an energetics and informatics whereby changes in energy gradients are the very marks, traces, and messages that constitute an individual. Thus individuation is achieved through varying intensive and extensive speeds characterizing different gestures. Hence we get both the Kittler refrain "There is no such thing as software," since there are always material relations at some scale of processing, and we also get an entirely new way of thinking about the material world.

The LSS shows how social scientists might move "from perception-centred accounts of experience to a broader understanding of sensibility as the concrete texture of experience across the board" (Hansen 2015, 48). LSS dethrones the human subject as the site of perception synthesis and begins to adumbrate ways that researchers might study the highly impersonal forms of being produced through massive data mining techniques. Speculative fiction more generally allows us to explore the expansion of the sensory confound that characterizes contemporary computational environments. Twenty-firstcentury media are radically overhauling the manner by which life is lived, as microtemporal biometric data circulate and are absorbed at rates well below and above the bandwidth of human consciousness.

One of the virtues of science fiction is its power to distance us from our everyday assumptions about cognition: "In science fiction narratives, cognition may fail because new technologies 'alter sense ratios or patterns of perception' so radically that there is no evident pathway from here to there; or because the sort of subjectivity that we take for granted has broken down; or because we encounter alien forms of sentience that are not commensurable with our own" (Shaviro 2015, 39). We see how the LSS speculative fiction allows us to consider the broad concept of sentience - a much broader concept than mere consciousness - so as to better understand the force of affect in what Shaviro calls "discognition" (against the cognitive image of thought and mind). This takes us into the realm of what Ian Bogost (2012) calls alien phenomenology or Roden (2015) calls dark phenomenology. We have to probe the dark recesses of sentience using new methods that do not force a particular sensory regime onto it; our task is to examine the darkness in the dark, with other kinds of sensors. 
The LSS pursues this objective by gradually becoming attuned to the pragmatic or heuristic at work "in sentience," a kind of rumbling inexact repetition that always mutates and engenders the next iteration. It is not about special cognitive skills but about an "overall sensibility" (Shaviro 2015, 85) or a kind of synesthesia that saturates the milieu. In this way, we shift our approach to track an impersonal sensation. Perception becomes a kind of causality in this world, a kind of dispersed touching whereby everything is in contact.

If the power of speculative fiction is to imagine otherwise in plausible ways, then the LSS triggers this crucial question: How can more-than-human research practices serve an expanded notion of justice that might counter the forces of a control society? This question is best answered by looking carefully at the way time is operationalized in any experimental apparatus. Twenty-first-century media bypass the slow time resolution of human perception and make material contact with the sensory continuum. The technical sensors now ubiquitous in every context are registering events at the microtemporal level, and these data are fed forward into the futures of human perception. The crucial thing here is that technology is no longer a surrogate for a human faculty or capacity but instead operates directly on the sensibility of the "total" environment that precedes and underlies our own corporeal phenomenal experience. The microcomputational sensing that feeds into current data gathering and predictive analytics must be seen as exposing the radical exteriority of experience.

What marks the LSS as radical in its findings, based somewhat comically on the unexpected flood, concerns this advancement in synchronizing data gathering and analytics. The LSS thus is exactly where our nightmares of capitalist exploitation can come to fruition: it offers a thought experiment in which we can begin to grapple with how the temporality of "digital insights" changes the way we do the social sciences. The LSS could easily become a nightmare site where we chase only the simultaneity of sensory solicitation and response, a goal that inevitably serves the "brutal functionalism" of marketing firms and culture industries (Hansen 2015, 58). But the LSS also offers a means for imagining and building a different sociological methodology. If we want to reclaim these digital insights for more creative efforts, so that computational sensing serves more than corporate interests, speculative fiction offers one way of engendering alternative futures.

\section{Works Cited}

Bogost, Ian. 2012. Alien Phenomenology, or What It's Like to Be a Thing. Minneapolis: University of Minnesota Press. 
Delanda, Manuel. 2011. Philosophy and Simulation: The Emergence of Synthetic Reason. New York: Bloomsbury.

Deleuze, Gilles, and Félix Guattari. 1987. A Thousand Plateaus: Capitalism and Schizophrenia. Translated by Brian Massumi. Minneapolis: University of Minnesota Press.

Dennett, Daniel. 1991. Consciousness Explained. Boston: Little, Brown.

Galloway, Alexander, and Eugene Thacker. 2007. The Exploit: A Theory of Networks. Minneapolis: University of Minnesota Press.

Hansen, Mark. 2015. Feed-Forward: On the Future of Twenty-First-Century Media. Chicago: University of Chicago Press.

Latour, Bruno. 2005. Reassembling the Social: An Introduction to Actor-NetworkTheory. Oxford: Oxford University Press.

Morton, Timothy. 2013. Hyperobjects: Philosophy and Ecology after the End of the World. Minneapolis: University of Minnesota Press.

Parisi, Luciana. 2016. "Automated Thinking and the Limits of Reason." In "Alternative Ontologies of Number: Rethinking the Quantitative in Computational Culture," edited by Elizabeth de Freitas, E. Ramon-Dixon, and P. Lather, special issue, Critical Methodologies - Cultural Studies 16, no. 5: 435-41.

Roden, David. 2015. Posthuman Life: Philosophy at the Edge of the Human. New York: Routledge.

Shaviro, Steven. 2015. Discognition. London: Repeater.

Stengers, Isabelle. 2000. Invention of Modern Science. Minneapolis: University of Minnesota Press. 